\title{
SPAN-WISE FLUCTUATING MHD CONVECTIVE FLOW OF A VISCO- ELASTIC FLUID THROUGH A POROUS MEDIUM IN A HOT VERTICAL CHANNEL WITH THERMAL RADIATION
}

\author{
K.D. SINGH \\ Applied Mathematics, Wexlow \\ Lower Kaithu, Shimla-171003, INDIA \\ E-mail: kdsinghshimla@gmail.com
}

\begin{abstract}
An unsteady mixed convection flow of a visco-elastic, incompressible and electrically conducting fluid in a hot vertical channel is analyzed. The vertical channel is filled with a porous medium. The temperature of one of the channel plates is considered to be fluctuating span-wise cosinusoidally, i.e., $T^{*}\left(y^{*}, z^{*}, t^{*}\right)=T_{1}+\left(T_{2}-T_{1}\right) \cos \left(\frac{\pi z^{*}}{d}-\omega^{*} t^{*}\right)$. A magnetic field of uniform strength is applied perpendicular to the planes of the plates. The magnetic Reynolds number is assumed very small so that the induced magnetic field is neglected. It is also assumed that the conducting fluid is gray, absorbing/ emitting radiation and non-scattering. Governing equations are solved exactly for the velocity and the temperature fields. The effects of various flow parameters on the velocity, temperature and the skin friction and the Nusselt number in terms of their amplitudes and phase angles are discussed with the help of figures.
\end{abstract}

Key words: magnetohydrodynamics (MHD), span-wise cosinusoidal, convective, porous medium, radiation.

\section{Introduction}

The flows of visco-elastic fluids through porous media are very important particularly in the fields of petroleum technology for the flow of oil through porous rocks, in chemical engineering for the purification and filtration processes and in the cases like drug permeation through human skin. The principles of this subject are very useful in recovering water for drinking and irrigation purposes. The knowledge of flows through porous media is also useful to study the movement of natural gas and water through the oil reservoirs. A number of studies have appeared in the literature where the porous medium is either bounded between parallel plates or bounded by flat plates. A detailed account of the applications of the convection flows through a porous media has been reported by Nield and Bejan [1]. Raptis [2] and Raptis and Perdikis [3] studied the unsteady free convection flow through porous medium bounded by infinite porous plates. Prasuma et al. [4] investigated an unsteady flow of a viscoelastic fluid through a porous medium between two impermeable parallel plates. Rajgopal [5] analyzed the Stoke's problem for a non-Netonian fluid. Pillai et al. [6] studied heat transfer in a viscoelastic boundary layer flow through a porous medium. Hossain and Takhar [7] studied the radiation effects on mixed convection along a vertical plate with uniform surface temperature.

The unsteady mixed convection hydromagnetic flows through a porous medium remained of considerable interest due to their occurrence in nature and in different applications in many branches of science and technology. Magnetohydrodynamic (MHD) is currently going through a period of great development and differentiation of subject matter. The mixed convection boundary layer flow of a nonNewtonian fluid in the presence of a magnetic field has wide range of application in nuclear engineering and industries. In astrophysical and geophysical studies, the MHD boundary layer flows of an electrically conducting fluid through porous media have also enormous applications. The interest in 
magnetohydrodynamic (MHD) convection with heat transfer is renewed due to its importance in the design of MHD generators, and accelerators in geophysics, in the design of underground water and energy storage systems, soil sciences, astrophysics and so on. Most of the industrial fluids are non-Newtonian and some of them are electrically conducting. Viscoelastic fluids, containing the viscous stresses as well as elastic responses in the constitutive relations exhibit different types of flow phenomenon. Materials such as, paints, adhesive, coal products, etc., are few examples of viscoelastic fluids. Moreover, bio-chemical materials (in liquid states) are also to be included in this class of fluids. Besides, the environmentally polluted material such as industrial wastes in liquid state may also be considered to be another kind of non-Newtonian fluids. Rajgopal et al. [8] studied oscillatory flow of an electrically conducting visco-elastic fluid over a stretching sheet in a saturated porous medium. Singh [9] obtained an exact solution of an oscillatory MHD flow in a channel filled with a porous medium. Rahman and Sarkar [10] analyzed the unsteady MHD flow of a dusty visco-elastic Oldroyd fluid under time varying body force through a rectangular channel. Singh and Singh [11] studied the MHD flow of a dusty visco-elastic (Oldroyd B- liquid) through a porous medium between two parallel plates inclined to the horizon. Attia and Ewis [12] investigated an unsteady MHD Couette flow with heat transfer of a visco-elastic fluid under exponentially decaying pressure gradient. Recently, Choudhary and Das [13] extended the problem of Makinde and Mhone [14] by taking into account the viscoelastic fluid. Singh [15] studied a viscoelastic mixed convection MHD oscillatory flow through a porous medium filled in a vertical channel. Recently, Singh [16] further studied the viscoelastic MHD oscillatory forced convection flow in a vertical channel with heat radiation.

There are many material processing industrial operations where due to high temperatures all the three modes of heat transportation such as conduction, convection and radiation occur together. The important areas in which thermal radiation heat transfer must be considered along with thermal convection heat transfer are direct flame impingement (DFI) furnace for rapid heating of metals in materials processing (Malikov et al. [17]), heating of a continuously moving load in the industrial radiant oven (Fedorov et al. [18]) and glass melting simulation (Lentes and Siedow [19]).

The high temperatures in majority of industrial processes may not be uniform everywhere in the processing plants. In other words, these high temperatures may not necessarily be constant. They may vary over a period of time and at the same time may be different at different locations. In view of this a few studies have been conducted by considering span-wise cosinusoidal temperatures of the surfaces. Singh [20] analyzed an unsteady free convection flow past a hot vertical porous plate with variable temperature. Singh and Chand [21] discussed an unsteady free convective MHD flow past a vertical porous plate with such a variation of the temperature. Sumathi et al. [22] also studied heat and mass transfer in an unsteady three dimensional mixed convection flow past an infinite vertical porous plate with cosinusoidally fluctuating temperature. Kumar and Singh [23] studied an unsteady MHD flow of radiating and reacting fluid past a vertical porous plate with cosinusoidally fluctuating temperature.

Hence, the purpose of the present work is to analyze the visco-elastic fluid flow in a vertical channel filled with the porous medium when the temperature of one of the channel plates varies both in space and time. A uniform transverse magnetic field is applied and heat due to radiation is taken into consideration. The magnetic Reynolds number is assumed to be small enough so that the induced magnetic field is neglected. The non-uniform temperature difference of the channel walls is high enough to induce thermal radiations. The partial differential equations are solved exactly and the final results for the velocity, temperature and the skin friction and rate of heat transfer in terms of their amplitude and phase angles are evaluated numerically for different values of the flow parameters. The findings are presented graphically and discussed in the last section of the paper.

\section{Basic equations}

In order to derive basic equations for the problem under consideration the following assumptions are made:

(i) The flow considered is unsteady and laminar. 
(ii) The fluid is a second order viscoelastic, incompressible, finitely conducting and with constant physical properties.

(iii) A magnetic field of uniform strength is applied transverse to the flow.

(iv) The magnetic Reynolds number is taken to be small enough so that the induced magnetic field is neglected.

(v) Hall effect, electrical and polarization effects are neglected.

(vi) It is also assumed that the conducting fluid is an optically-thin gray, absorbing/ emitting radiation and non-scattering gas.

Under these assumptions the equations of continuity, motion and energy governing an unsteady hydromagnetic (MHD) convective flow through a porous medium are

$$
\begin{aligned}
& \nabla . \boldsymbol{V}=0 \\
& \frac{\partial \boldsymbol{V}}{\partial t}+(\boldsymbol{V} . \nabla) \boldsymbol{V}=\nabla . \exists+\frac{\vartheta_{1}}{\boldsymbol{K}^{*}} \boldsymbol{V}+\frac{1}{\rho}(\boldsymbol{J} \times \boldsymbol{B})+\boldsymbol{F}, \\
& \rho C_{p}\left[\frac{\partial T^{*}}{\partial t^{*}}+(\boldsymbol{V} . \nabla) T^{*}\right]=k \nabla^{2} T^{*}-\nabla q^{*} .
\end{aligned}
$$

On the right hand side of Eq.(2.2) the last term $\boldsymbol{F}\left(=g \beta T^{*}\right)$ accounts for the force due to buoyancy and the second last term is the Lorentz force due to the magnetic field $\boldsymbol{B}$ and is given by

$$
\boldsymbol{J} \times \boldsymbol{B}=\sigma(\boldsymbol{V} \times \boldsymbol{B}) \times \boldsymbol{B}
$$

In the first term on the right hand side of Eq.(2.2), $\exists$ is the Cauchy stress tensor and the constitutive equation derived by Coleman and Noll [24] for an incompressible homogeneous fluid of second order is

$$
\exists=-p_{1} I+\mu_{1} A_{1}+\mu_{2} A_{2}+\mu_{3} A_{1}^{2} .
$$

Here $-p_{1} I$ is the interdeterminate part of the stress due to constraint of incompressibility, $\mu_{l}, \mu_{2}$ and $\mu_{3}$ are the material constants describing viscosity, elasticity and cross-viscosity, respectively. The kinematic $A_{1}$ and $A_{2}$ are the Rivlin Ericson constants defined as

$$
A_{l}=(\nabla V)+(\nabla V)^{T}, \quad A_{2}=\frac{d A_{1}}{d t}+(\nabla V)^{T} A_{l}+A_{l}(\nabla V)
$$

where $\nabla$ denotes the gradient operator and $d / d t$ the material time derivative. According to Markovitz and Coleman [25] the material constants $\mu_{1}, \mu_{3}$ are taken as positive and $\mu_{2}$ as negative. The modified pressure $p^{*}=p^{\prime}-\frac{\rho}{2}|\boldsymbol{\Omega} \times \boldsymbol{R}|^{2}$, where $\boldsymbol{R}$ denotes the position vector from the axis of rotation, $p^{\prime}$ denotes the fluid pressure, $\boldsymbol{J}$ is the current density and all other quantities have their usual meanings and have been defined in the text. 


\section{Formulation of the problem}

In the present analysis, we consider an unsteady flow of a viscoelastic (second order) incompressible and electrically conducting fluid bounded by two infinite insulated vertical plates distance ' $d$ ' apart. A coordinate system is chosen such that the the $X^{*}$-axis is oriented upward along the centerline of the channel and the $Y^{*}$-axis is taken perpendicular to the planes of the plates lying in $y^{*}= \pm \frac{d}{2}$ planes. The non-uniform temperature of the plate at $y^{*}=+\frac{d}{2}$ is assumed to be varying in both space and time as

$$
T^{*}\left(y^{*}, z^{*}, t^{*}\right)=T_{1}+\left(T_{2}-T_{1}\right) \cos \left(\frac{\pi z^{*}}{d}-\omega^{*} t^{*}\right) \text {. }
$$

A transverse magnetic field of uniform strength $\boldsymbol{B}\left(0, B_{0}, 0\right)$ is also applied along the $Y^{*}$-axis. Since the channel plates are assumed impermeable, the equation of continuity integrates to $v^{*}=0$. Therefore, the velocity may reasonably be assumed with its components along $x^{*}, y^{*}, z^{*}$ directions as $\boldsymbol{V}\left(u^{*}, 0,0\right)$. Since the vertical parallel plates are infinite along the $X^{*}$-axis, therefore all physical quantities except the pressure are independent of $x^{*}$. A schematic diagram of the physical problem considered is presented in Figs $1 \mathrm{a}$ and $1 \mathrm{~b}$ where Fig.1b shows the span-wise cosinusoidal fluctuating temperature of the channel plate variation at $y^{*}=+\frac{d}{2}$.

$$
y^{*}=-\frac{d}{2}, \quad y^{*}=+\frac{d}{2} .
$$

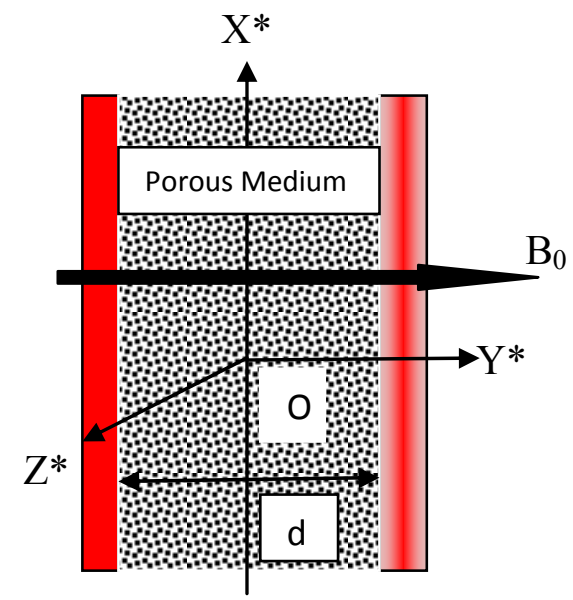

Fig.1a. Hot vertical channel.4

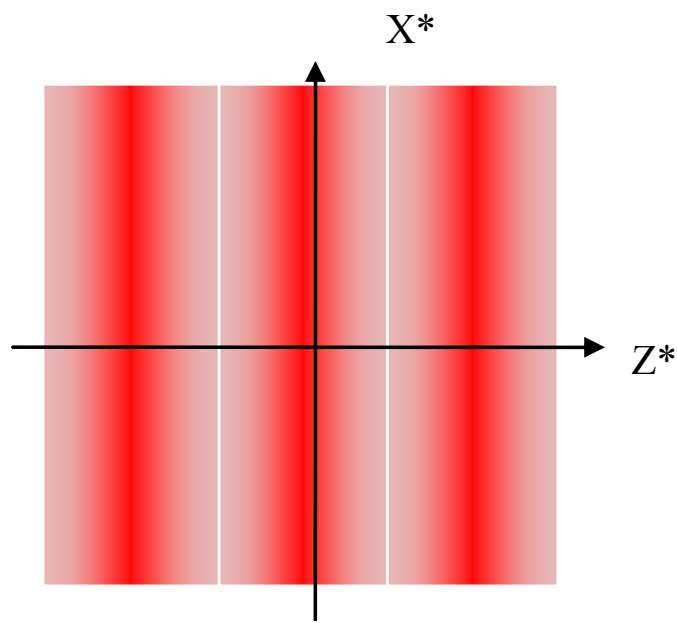

Fig.1b. Span-wise cosinusoidal plate temperature.

Using the velocity and the magnetic field distribution as stated above the magnetohydrodynamic (MHD) flow through a porous medium in the channel is governed by the following Cartesian equations 


$$
\begin{aligned}
& \frac{\partial u^{*}}{\partial t^{*}}=-\frac{1}{\rho} \frac{\partial p^{*}}{\partial x^{*}}+\vartheta_{1}\left(\frac{\partial^{2} u^{*}}{\partial y^{* 2}}+\frac{\partial^{2} u^{*}}{\partial z^{* 2}}\right)+\vartheta_{2} \frac{\partial}{\partial t^{*}}\left(\frac{\partial^{2} u^{*}}{\partial y^{* 2}}+\frac{\partial^{2} u^{*}}{\partial z^{* 2}}\right)+ \\
& -\frac{\sigma B_{0}^{2}}{\rho} u^{*}-\frac{\vartheta_{1} u^{*}}{K^{*}}+g \beta\left(T^{*}-T_{1}\right), \\
& \frac{\partial T^{*}}{\partial t^{*}}=\frac{k}{\rho c_{p}}\left(\frac{\partial^{2} T^{*}}{\partial y^{* 2}}+\frac{\partial^{2} T^{*}}{\partial z^{* 2}}\right)-\frac{1}{\rho c_{p}} \frac{\partial q^{*}}{\partial y^{*}}
\end{aligned}
$$

where $\rho$ is the density, $\mu$ is the viscosity, $\vartheta_{1}$ is the kinematic viscosity, $\vartheta_{2}$ is the viscoelasticity, $p^{*}$ is the modified pressure, $t^{*}$ is the time, $\sigma$ is the electric conductivity, $g$ is the acceleration due to gravity, $\beta$ is the coefficient of volume expansion, $K^{*}$ is the permeability of the porous medium, $k$ is the thermal conductivity, $c_{p}$ is the specific heat at constant pressure and $p^{*}$ is the pressure.

The boundary conditions for the problem are

$$
\begin{aligned}
& y^{*}=-\frac{d}{2}: \quad u^{*}=0, \quad T^{*}=T_{1}, \\
& y^{*}=\frac{d}{2}: \quad u^{*}=0, \quad T^{*}=T_{1}+\left(T_{2}-T_{1}\right) \cos \left(\frac{\pi z^{*}}{d}-\omega^{*} t^{*}\right)
\end{aligned}
$$

where $\omega^{*}$ is the frequency of oscillations.

The last term in Eq.(2.3) stands for heat due to radiation in the $y^{*}$-direction. Following Raptis et al. [26] the local radiant for the case of an optically thin gray, absorbing/ emitting radiation and non-scattering gas is expressed by

$$
\frac{\partial q^{*}}{\partial y^{*}}=4 a^{\prime} \sigma^{\prime}\left(T^{* 4}-T_{1}^{4}\right)
$$

where $a$ is the mean absorption coefficient and $\sigma^{\prime}$ is the Stefan- Boltzmann constant. We assume that the temperature differences within the flow are sufficiently small such that $T^{* 4}$ may be expressed as a linear function of the temperature. This is accomplished by expanding $T^{* 4}$ in a Taylor series about $T_{1}$ and neglecting higher order terms, thus

$$
T^{* 4} \cong 4 T_{1}^{* 3} T^{*}-3 T_{1}^{4}
$$

Substituting Eq.(3.7) into Eq.(3.6) and simplifying, we obtain

$$
\frac{\partial q^{*}}{\partial y^{*}}=16 a^{\prime} \sigma^{\prime} T_{1}^{3}\left(T^{*}-T_{1}\right) \text {. }
$$

The substitution of Eq.(3.8) into the energy Eq.(3.3) for the heat due to radiation, we get 


$$
\frac{\partial T^{*}}{\partial t^{*}}=\frac{k}{\rho c_{p}}\left(\frac{\partial^{2} T^{*}}{\partial y^{* 2}}+\frac{\partial^{2} T^{*}}{\partial z^{* 2}}\right)-\frac{16 a^{\prime} \sigma^{\prime} T_{1}^{3}}{\rho c_{p}}\left(T^{*}-T_{1}\right) .
$$

Now introducing the following non-dimensional quantities

$$
x, y, z=\frac{x^{*}, y^{*}, z^{*}}{d}, \quad t=\omega^{*} t^{*}, \quad \omega=\frac{\omega^{*} d^{2}}{\vartheta}, \quad u=\frac{u^{*}}{U}, \quad \theta=\frac{T^{*}-T_{1}}{T_{2}-T_{1}}, \quad p=\frac{d p^{*}}{\mu U},
$$

in Eqs (3.2), (3.9), (3.4) and (3.5) we obtain governing equations and the boundary conditions in a dimensionless form as

$$
\begin{aligned}
& \omega \frac{\partial u}{\partial t}=-\frac{\partial p}{\partial x}+\left(\frac{\partial^{2} u}{\partial y^{2}}+\frac{\partial^{2} u}{\partial z^{2}}\right)+\gamma \omega \frac{\partial}{\partial t}\left(\frac{\partial^{2} u}{\partial y^{2}}+\frac{\partial^{2} u}{\partial z^{2}}\right)-\left(M^{2}+K^{-1}\right) u+\operatorname{Gr} \theta \\
& \omega \operatorname{Pr} \frac{\partial \theta}{\partial t}=\left(\frac{\partial^{2} \theta}{\partial y^{2}}+\frac{\partial^{2} \theta}{\partial z^{2}}\right)-N^{2} \theta
\end{aligned}
$$

with boundary conditions

$$
\begin{aligned}
& u=0, \quad \theta=0, \quad \text { at } \quad y=-\frac{1}{2}, \\
& u=0, \quad \theta=\cos (\pi z-t), \quad \text { at } \quad y=\frac{1}{2}
\end{aligned}
$$

where $\mathrm{Gr}=\frac{g \beta\left(T_{2}-T_{1}\right) d^{2}}{\vartheta U}$ is the Grashof number,

$$
\begin{aligned}
& \operatorname{Pr}=\frac{\mu c_{p}}{k}, \text { is the Prandtl number, } \\
& M=B_{0} d \sqrt{\frac{\sigma}{\mu}}, \text { is the Hartmann number, } \\
& K=\frac{K^{*}}{d^{2}}, \text { is the permeability of the porous medium, } \\
& N=4 d \sqrt{\frac{a^{\prime} \sigma^{\prime} T_{1}^{3}}{k}} \text {, is the radiation parameter, } \\
& \gamma=\frac{\vartheta_{2}}{d^{2}} \text { is the visco-elastic parameter }
\end{aligned}
$$

where $U$ is the mean flow velocity. 


\section{Solution of the problem}

In order to obtain the solution of this unsteady problem it is convenient to adopt complex variable notations for velocity, temperature and pressure. The real part of the solution will have physical significance. Thus, we write velocity, temperature and pressure as

$$
u(y, z, t)=u_{0}(y) e^{i(\pi z-t)}, \quad \theta(y, z, t)=\theta_{0}(y) e^{i(\pi z-t)}, \quad-\frac{\partial p}{\partial x}=A e^{i(\pi z-t)}
$$

where $A$ is a constant.

The boundary conditions in Eqs (3.13) and (3.14) can be written in complex notations as

$$
\begin{aligned}
& u=0, \quad \theta=0, \quad \text { at } \quad y=-\frac{1}{2}, \\
& u=0, \quad \theta=e^{i(\pi z-t)}, \quad \text { at } \quad y=\frac{1}{2} .
\end{aligned}
$$

Substituting expressions (4.1) into Eqs (3.11) and (3.12), we get

$$
\begin{aligned}
& (1-i \omega \gamma) \frac{d^{2} u_{0}}{d y^{2}}-m^{2} u_{0}=-A-\operatorname{Gr} \theta_{0} \\
& \frac{d^{2} \theta_{0}}{d y^{2}}-n^{2} \theta_{0}=0
\end{aligned}
$$

where $m=\sqrt{\pi^{2}+M^{2}+K^{-1}-i \omega\left(1+\gamma \pi^{2}\right)}$ and $n=\sqrt{\left(\pi^{2}+N^{2}-i \omega \operatorname{Pr}\right)}$

with transformed boundary conditions

$$
\begin{aligned}
& u_{0}=0, \quad \theta_{0}=0 \quad \text { at } \quad y=-\frac{1}{2}, \\
& \mathrm{u}_{0}=0, \quad \theta_{0}=1 \quad \text { at } \quad y=\frac{1}{2} .
\end{aligned}
$$

The ordinary differential Eqs (4.4) and (4.5) are solved under boundary conditions Eqs (4.6) and (4.7) and solutions for the velocity and the temperature fields are obtained, respectively, as

$$
u(y, z, t)=\left\{\frac{A}{m^{2}}\left(1-\frac{\cosh \frac{m}{l} y}{\cosh \frac{m}{2 l}}\right)+\frac{\mathrm{Gr}}{\left(l^{2} n^{2}-m^{2}\right)}\left(\frac{\sinh \frac{m}{l}\left(y+\frac{l}{2}\right)}{\sinh \frac{m}{l}}-\frac{\sinh n\left(y+\frac{l}{2}\right)}{\sinh n}\right)\right\} e^{i(\pi z-t)}
$$




$$
\theta(y, z, t)=\left\{\frac{\sinh n\left(y+\frac{1}{2}\right)}{\sinh n}\right\} e^{i(\pi z-t)}
$$

where $l=\sqrt{1-i \omega \gamma}$.

From the velocity field in Eq.(4.8) we can obtain the skin-friction at the left wall, $\tau_{L}$, in terms of its amplitude $|F|$ and the phase angle $\varphi$ as

$$
\tau_{L}=\left(\frac{\partial u_{0}}{\partial y}\right)_{y=-\frac{1}{2}}|F| \cos (\pi z-t+\varphi)
$$

where

$$
F_{r}+i F_{i}=\frac{A}{l m} \tanh \frac{m}{2 l}+\frac{\mathrm{Gr}}{\left(l^{2} n^{2}-m^{2}\right)}\left(\frac{\frac{m}{l}}{\sinh \frac{m}{l}}-\frac{n}{\sinh n}\right)
$$

The amplitude and the phase angle of the skin-friction $\tau_{L}$ are respectively given by

$$
|F|=\sqrt{F_{r}^{2}+F_{i}^{2}}, \quad \text { and } \quad \varphi=\tan ^{-1}\left(\frac{F_{i}}{F_{r}}\right) .
$$

From the temperature field given in Eq.(4.9) the heat transfer coefficient $\mathrm{Nu}$ (Nusselt number) in terms of its amplitude and the phase angle can be obtained as

$$
\mathrm{Nu}=\left(\frac{\partial \theta}{\partial y}\right)_{y=-\frac{1}{2}}=|H| \cos (\pi z-t+\psi)
$$

where $H_{r}+i H_{i}=\frac{n}{\sinh n}$.

The amplitude $|H|$ and the phase angle $\psi$ of the heat transfer coefficient $\mathrm{Nu}$ (Nusselt number) are given by

$$
|H|=\sqrt{H_{r}^{2}+H_{i}^{2}} \quad \text { and } \quad \psi \psi=\tan ^{-1}\left(\frac{H_{i}}{H_{r}}\right) \text { respectively. }
$$

\section{Discussion of the results}

The hydrodynamic convective flow of a viscoelastic fluid in an infinite vertical channel filled with a porous medium is analyzed when the non-uniform temperature of one of the channel plates varies both in space and time. In the presence of a transverse magnetic field an exact solution of the problem is obtained. 
The velocity field, temperature field and the shear stress and rate of heat transfer in terms of their amplitudes and phase angles are evaluated numerically for different sets of the values of the viscoelastic parameter $\gamma$, Grashof number Gr, permeability of the porous medium $K$, Prandtl number Pr, radiation parameter $N$, pressure gradient $A$ and the frequency of oscillations $\omega$. These numerical values are then shown graphically to assess the effect of each parameter.

Figure 2 illustrates the variation of velocity over the channel width with the increasing flow parameters. The sets of parameter values shown by curves in Fig.2 are listed in Tab.1.The dotted (.....) curve I corresponds to the viscous (Newtonian) fluid i.e., $\gamma=0$. To assess the effect of each of the parameters each curve is compared with the basic dashed (----) curve II. It is observed from this figure that the velocity decreases (curves II \& III) with the increasing viscoelastic parameter $\gamma$ (Non-Newtonian). The variation of the velocity with the increasing Grashof number Gr is presented by curves II \& IV $(\mathrm{Gr}=1 \& 5)$. It is observed from these curves that the velocity increases with $\mathrm{Gr}$ and the maximum of the parabolic velocity profiles shifts toward right half of the channel due to the greater buoyancy force in this part of the channel due to the presence of a hotter plate and heat is transferred from the hot plate to the fluid and consequently the buoyancy force enhances the flow velocity further.

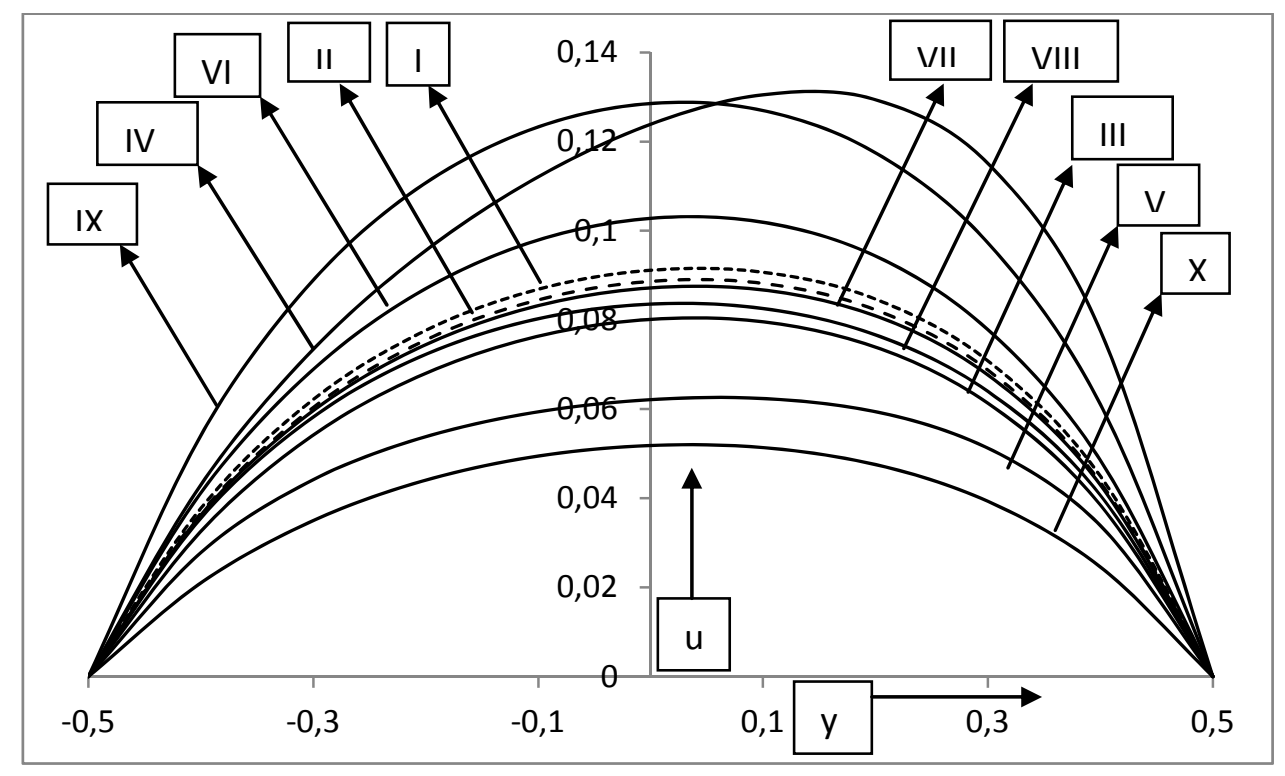

Fig.2. Velocity profiles for $z=0.5$ and $t=\pi / 2$.

Table 1. Sets parameter values plotted in Fig.2.

\begin{tabular}{|cccccccll|}
\hline$\gamma$ & Gr & $M$ & $K$ & $\operatorname{Pr}$ & $N$ & $A$ & $\omega$ & Curve \\
\hline 0 & 1 & 2 & 0.2 & 0.7 & 1 & 2 & 1 & I (...) \\
\hline 0.2 & 1 & 2 & 0.2 & 0.7 & 1 & 2 & 1 & II(---) \\
\hline 0.5 & 1 & 2 & 0.2 & 0.7 & 1 & 2 & 1 & III \\
\hline 0.2 & 5 & 2 & 0.2 & 0.7 & 1 & 2 & 1 & IV \\
\hline 0.2 & 1 & 4 & 0.2 & 0.7 & 1 & 2 & 1 & V \\
\hline 0.2 & 1 & 2 & 1.0 & 0.7 & 1 & 2 & 1 & VI \\
\hline 0.2 & 1 & 2 & 0.2 & 7.0 & 1 & 2 & 1 & VII \\
\hline 0.2 & 1 & 2 & 0.2 & 0.7 & 5 & 2 & 1 & VIII \\
\hline 0.2 & 1 & 2 & 0.2 & 0.7 & 1 & 3 & 1 & IX \\
\hline 0.2 & 1 & 2 & 0.2 & 0.7 & 1 & 2 & 5 & $\mathrm{X}$ \\
\hline
\end{tabular}


The effects of the magnetic field on the velocity field are depicted by curves II \& V. It is observed that the velocity decreases as the Hartmann number $M$ increases from 2 to 4 . This means that the increasing Lorentz force due to the increasing magnetic field strength resists the flow. Curves II \& VI show the variation of the velocity with the permeability of the porous medium $K$. It is found that the velocity increases with the increasing values of $K$ from 0.2 to 1 . It means physically that the resistance posed by the porous matrix decreases with the increasing permeability of the porous medium. A comparison of curves II \& VII reveals that the velocity decreases with the increasing Prandtl number Pr. The two values of the Prandtl number chosen correspond to most common fluids air $(\operatorname{Pr}=0.7)$ and water $(\operatorname{Pr}=7)$. This means that the velocity in air $(\mathrm{Pr}=0.7)$ is greater than in the case of water $(\mathrm{Pr}=7)$. Curves II \& VIII $(\mathrm{N}=1 \& 5)$ depict that the velocity decreases with the increasing radiation parameter $N$. It is evident from curves II \& IX (A $=2 \&$ 3 ) that the velocity goes on increasing with the increasing favorable pressure gradient $A(>0)$. The effect of the frequency of oscillations $\omega$ on the velocity is exhibited by curves II \& X ( $\omega=1 \& 5)$. It is noticed that velocity decreases with the increasing frequency $\omega$.

The skin-friction amplitude $|F|$ and the phase angle $\varphi$ are presented in Figs 3 and 4 respectively against $\omega$ the frequency of oscillations for sets of parameter values listed in Tab.2. A comparison of curves III, V, and VIII with the dotted curve I (---) in Fig.3 reveals that the amplitude increases with the increase of the Grashof number Gr, permeability of the porous medium $K$ and the pressure gradient $A$. But comparing the rest of the curves II, IV, VI, and VII with the dotted curve I (---) shows that $|F|$ decreases with the increasing viscoelastic parameter $\gamma$, Hartmann number M, Prandtl number Pr, and radiation parameter $N$. It is also noticed that with the increasing frequency of oscillation $\omega$ the skin-friction amplitude $|F|$ goes on decreasing. Similarly, in Fig.4 comparing curves II, III, V with the dotted curve I (---) it is noticed that the phase angle $\varphi$ of the skin-friction increases with the increase of the viscoelastic parameter $\gamma$, Grashof number Gr and permeability of the porous medium $K$. However, from the comparison of curves IV, VI, VII and VIII with the dotted curve I (---) it is found that the phase angle $\varphi$ decreases with the increase of the Hartmann number M, Prandtl number Pr, radiation parameter $N$, and the pressure gradient $A$. It is also inferred that there is always a phase lead and this lead increases with the increasing frequency of oscillations $\omega$.

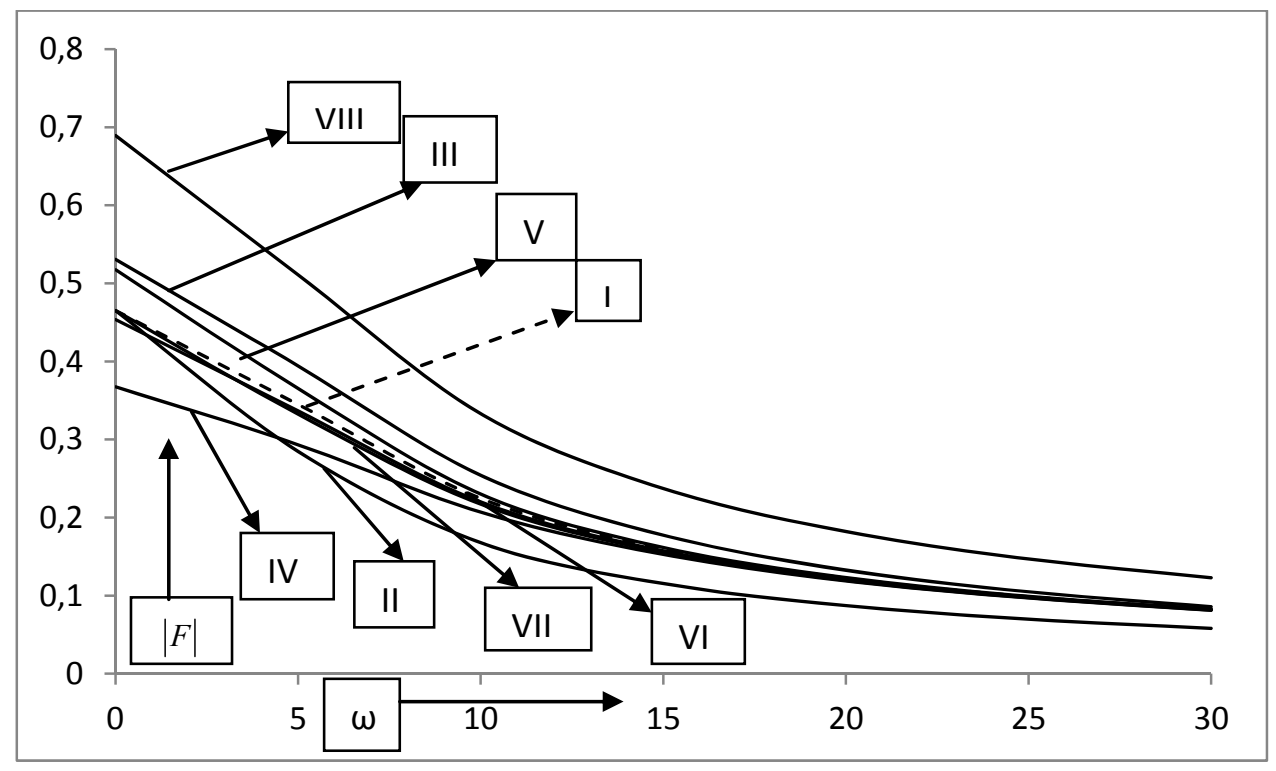

Fig.3. Amplitude of skin friction. 


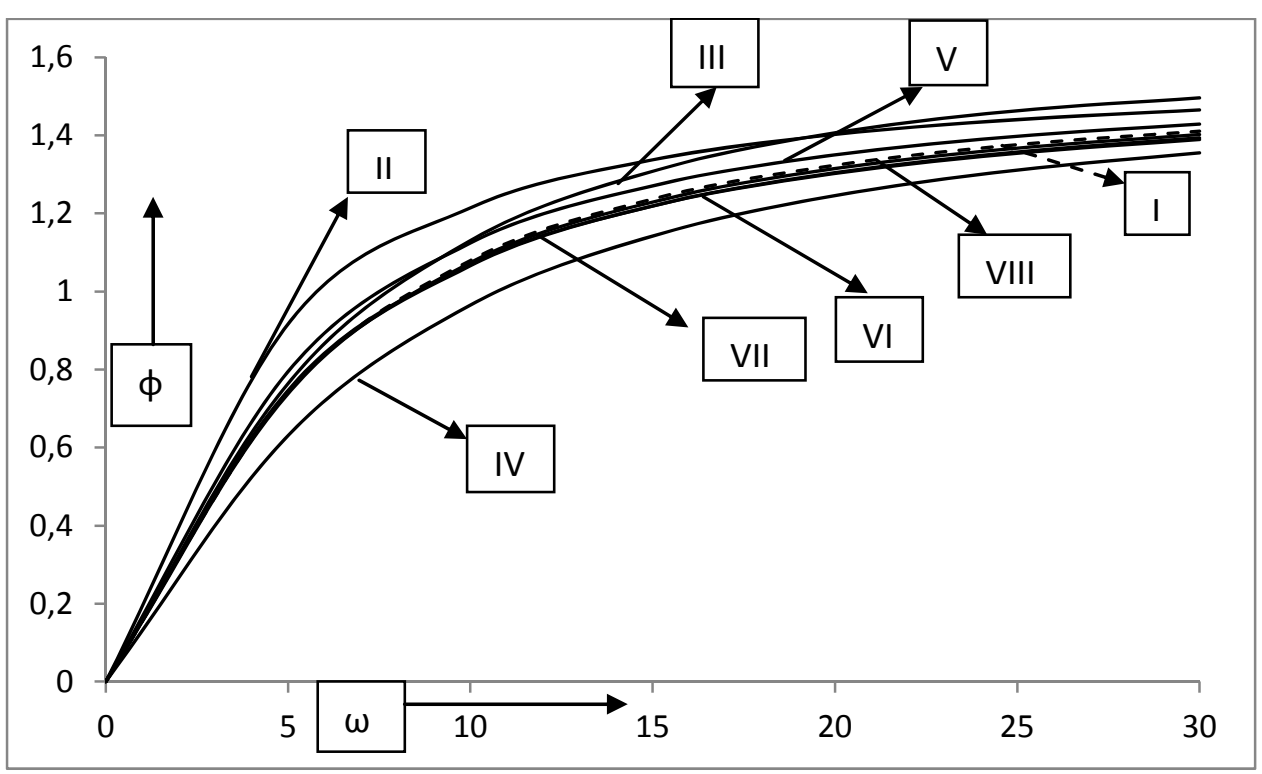

Fig.4. Phase angle of skin friction.

Table 2. Sets of parameter values plotted in Figs 3 and 4.

\begin{tabular}{|llllllll|}
\hline$\gamma$ & $\mathrm{Gr}$ & $M$ & $K$ & $\operatorname{Pr}$ & $N$ & $A$ & Curve \\
0.2 & 1 & 2 & 0.2 & 0.7 & 1 & 2 & $\mathrm{I}(---)$ \\
0.3 & 1 & 2 & 0.2 & 0.7 & 1 & 2 & $\mathrm{II}$ \\
0.2 & 5 & 2 & 0.2 & 0.7 & 1 & 2 & $\mathrm{III}$ \\
0.2 & 1 & 4 & 0.2 & 0.7 & 1 & 2 & $\mathrm{IV}$ \\
0.2 & 1 & 2 & 1.0 & 0.7 & 1 & 2 & $\mathrm{~V}$ \\
0.2 & 1 & 2 & 0.2 & 7.0 & 1 & 2 & $\mathrm{VI}$ \\
0.2 & 1 & 2 & 0.2 & 0.7 & 5 & 2 & $\mathrm{VII}$ \\
0.2 & 1 & 2 & 0.2 & 0.7 & 1 & 3 & $\mathrm{VIII}$ \\
& & & & & & & \\
\hline
\end{tabular}

The variations of the temperature with the Prandtl number Pr, radiation parameter $N$ and the frequency of oscillations $\omega$ are shown in Fig.5. It is obvious from this figure that the temperature decreases with the increase of all these parameters. 


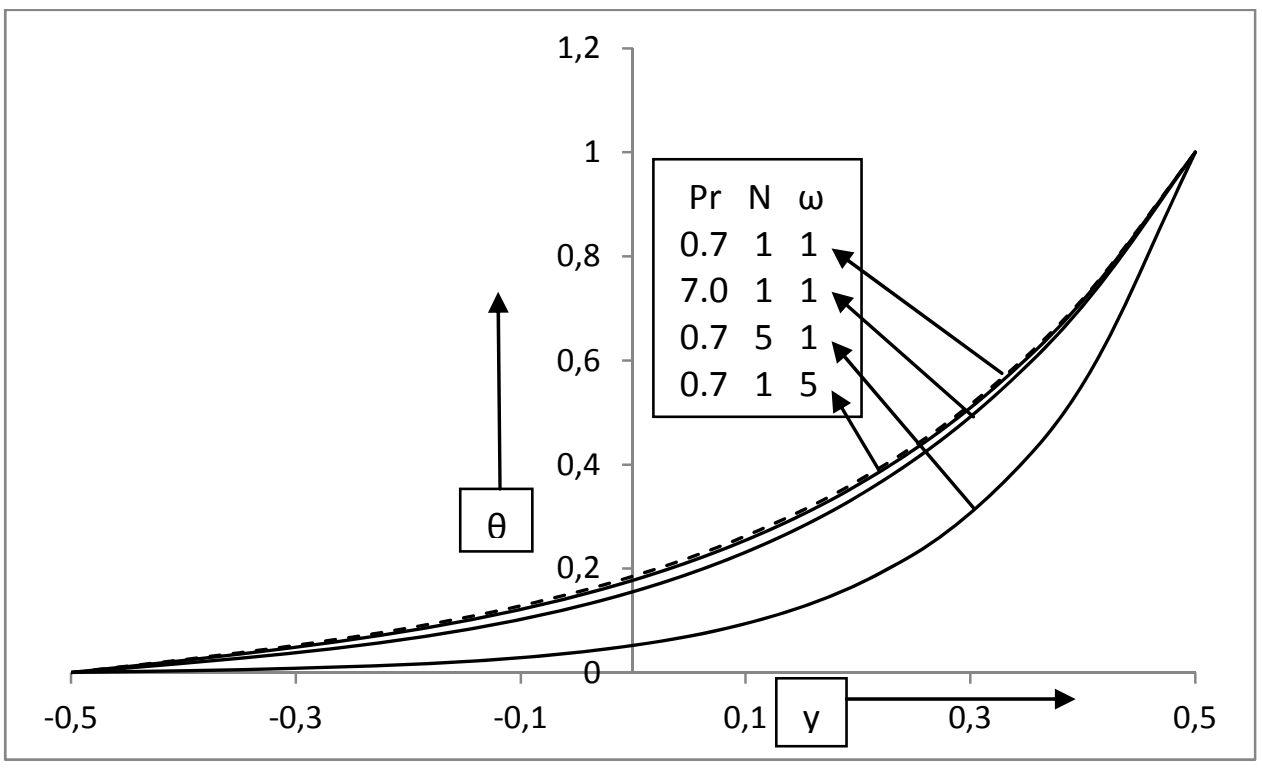

Fig.5. Temperature profiles for $z=0.5$ and $t=\pi / 2$.

The amplitude $|H|$ and the phase angle of the rate of heat transfer against the frequency of oscillations $\omega$ are illustrated in Figs 6 and 7, respectively. It is evident from Fig.6 that the amplitude $|H|$ decreases with the increase of the Prandtl number Pr and the radiation parameter $N$. It is also obvious that the amplitude $|H|$ goes on decreasing with the increasing frequency $\omega$ of oscillations for increased $\operatorname{Pr}$ but it remains almost constant for the increased radiation parameter $N$. The amplitude in the case of water $(\operatorname{Pr}=7)$ is far less than that in the case of air $(\operatorname{Pr}=0.7)$. Figure 7 shows that with increasing oscillations $\omega$ the phase angle $\psi$ of the heat transfer coefficient, $\mathrm{Nu}$, oscillates between the phase lag and the phase lead. There always remains a phase lead with increasing radiation parameter which varies linearly with increasing frequency $\omega$ of oscillations.

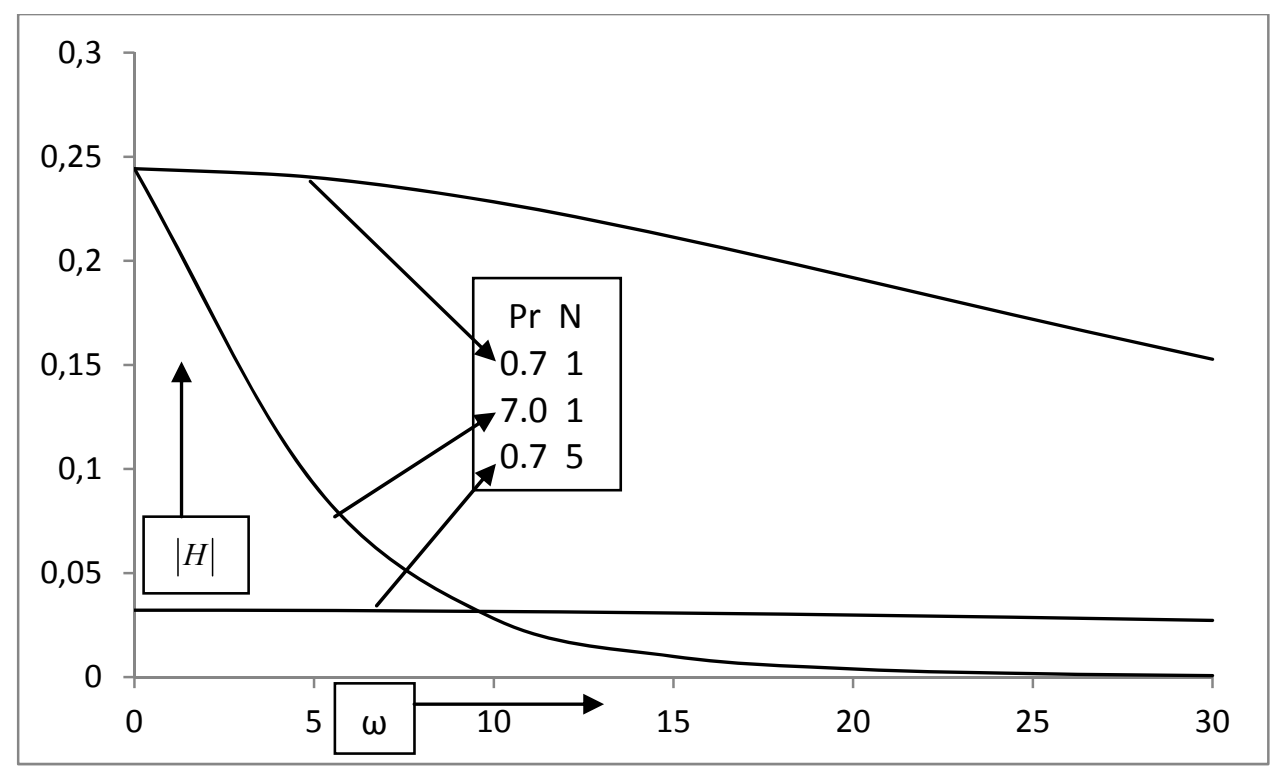

Fig.6. Amplitude of the Nusselt number. 


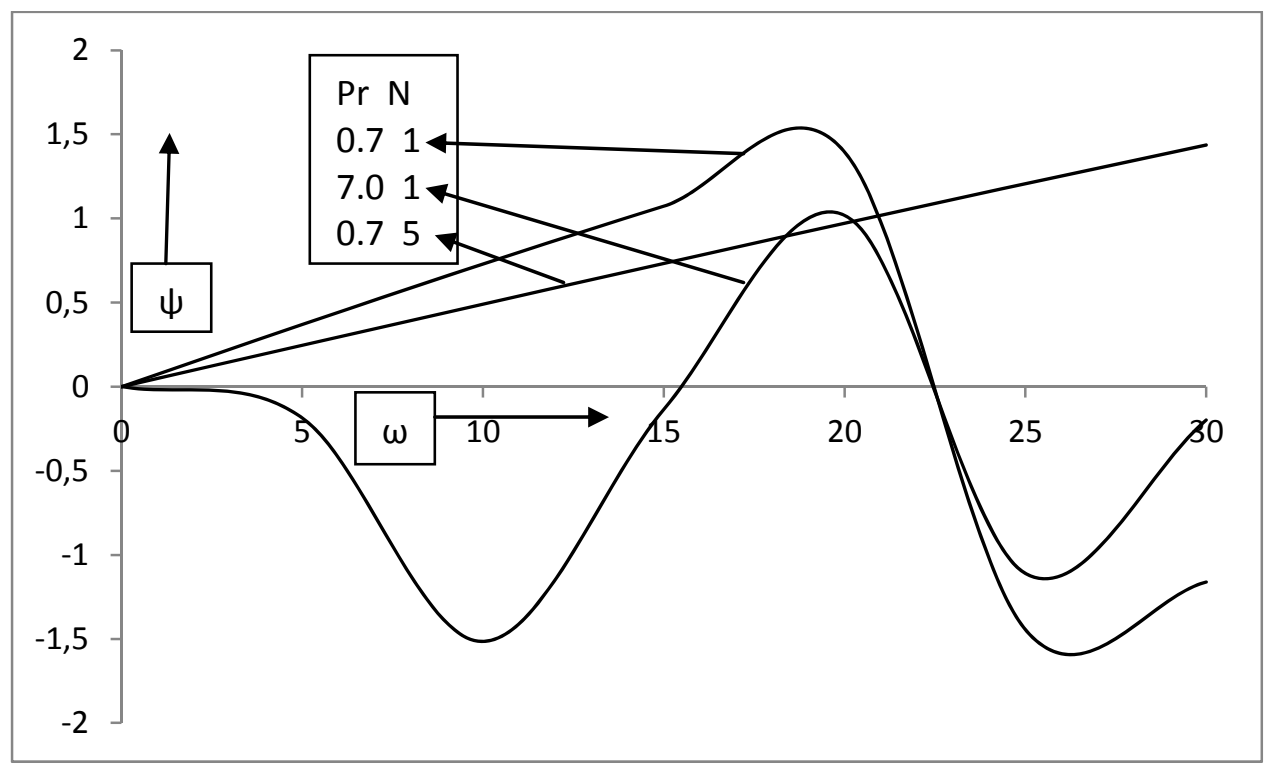

Fig.7. Phase angle of the Nusselt number.

\section{Conclusions}

The problem of an oscillatory MHD convection flow through the porous medium bounded by two infinite vertical porous plates is analyzed. A magnetic field of uniform strength is applied perpendicular to the plates of the channel. The temperature of one of the channel plates varies span-wise cosinusoidally. The following conclusions are made:-

- The velocity increases with the increasing Grashof number $\mathrm{Gr}$ or permeability of the porous medium $K$ or the pressure gradient $A$.

- The velocity decreases with the increasing viscoelstic parameter $\gamma$ or Hartmann number M or Prandtl number Pr or radiation parameter $N$ or the frequency of oscillations $\omega$.

- The amplitude of the skin-friction increases due to the increase of all those parameters because of which velocity increases, i.e., the Grashof number Gr or permeability of the porous medium $K$ or the pressure gradient $A$.

- Similarly, the skin-friction amplitude decreases due to the increase of parameters which lead to a decrease of velocity.

- The phase angle increases with the increasing viscoelstic parameter $\gamma$, Grashof number Gr or permeability of the porous medium $K$.

- Similarly the phase angle decreases with the increasing Hartmann number M or Prandtl number Pr or radiation parameter $N$ or pressure gradient $A$.

- There is always a phase lead.

- The temperature decreases with the Prandtl number Pr, radiation parameter $N$ and frequency of oscillations $\omega$.

- The amplitude $|H|$ of the rate of heat transfer decreases with the increasing Prandtl number Pr, radiation parameter $N$.

- The phase angle oscillates between the phase lead and phase lag for the increasing $\omega$.

\section{Nomenclature}

$a^{\prime}-$ means absorption coefficient

$A$ - constant 
$B_{0}$ - uniform magnetic field, [Tesla]

$c_{p}$ - specific heat at constant pressure, $\left[\mathrm{Jkg}^{-1} \mathrm{~K}^{-1}\right]$

$d$ - distance between plates

$|F|$ - amplitude of skin-friction

$g$ - acceleration due to gravity, $\left[\mathrm{m} / \mathrm{s}^{2}\right]$

Gr - Grashof number [-]

$|H|$ - amplitude of rate of heat transfer

$k$ - thermal conductivity, $\left[\mathrm{Wm}^{-1} \mathrm{~K}^{-1}\right]$

$K$ - permeability of the porous medium, [-]

$M$ - Hartmann number, [-]

$N$ - radiation parameter, [-]

Pr - Prandtl number, [-]

$q^{*}$ - radiative heat flux, $\left[\mathrm{Wm}^{-2}\right]$

$t$ - time, $[s]$

$T^{*}$ - fluid temperature, $[K]$

$T_{1}, T_{2}$ - constant temperatures

$U$ - mean axial velocity, $[\mathrm{m} / \mathrm{s}]$

$u^{*}$ - fluid velocity in the $x^{*}$-direction, $[\mathrm{m} / \mathrm{s}]$

$u, v, w$ - velocity components along the $x, y, z$-axis

$x, y, z$ - variables along the $X, Y, Z$-directions

$\beta$ - coefficient of thermal expansion, $\left[K^{-1}\right]$

$\gamma$ - viscoelastic parameter, [-]

$\theta_{0}-$ mean non-dimensional temperature

$\mu$ - viscosity

$\rho$ - fluid density, $\left[\mathrm{kgm}^{-3}\right]$

$\sigma$ - electrical conductivity, $\left[\mathrm{Sm}^{-1}\right]$

$\sigma^{\prime}-$ Stefan Boltzmann constant, $\left[\mathrm{Wm}^{-2} \mathrm{~K}^{-4}\right]$

$\tau_{L}-$ skin-friction at the left wall

$\vartheta_{1}$ - kinematic viscosity, $\left[\mathrm{m}^{2} \mathrm{~s}^{-1}\right]$

$\vartheta_{2}$ - viscoelasticity

$\varphi$ - phase angle of skin-friction

$\psi$ - phase angle of heat transfer

$\omega$ - frequency of oscillations

\section{Superscripts}

* - superscript for dimensional quantities

\section{References}

[1] Nield D.A. and Bejan A. (2006): Convection in Porous Media. - New York: Springer.

[2] Raptis A.A. (1983): Unsteady free convection flow through a porous medium. - Int. J. Engineering. Sci., vol.21, pp.345.

[3] Raptis A.A. and Perdikis C.P. (1985): Oscillatory flow through a porous medium by the presence of free convective flow. - Int. J. Engineering. Sci., vol.23, pp.51-55.

[4] Parsuma T.G., Murthy M.V.R., Ramachryulu N.C.P. and Rao G.V. (2010): Unsteady flow of a viscoelastic fluid through a porous media between two impermeable parallel plates. - J. of Emerging Trends in Engineering and Applied Sciences, vol.1, No.2, pp.220-224.

[5] Rajgopal K.R. (1983): On Stoke’s problem for non-Netonian fluid. - Acta Mech., vol.48 pp.223-239.

[6] Pillai K.M.C., Sai K.S., Swamy N.S., Natraja H.R., Tiwari S.B. and Rao B.N. (2004): Heat transfer in a viscoelastic boundary layer flow through porous medium. - Comput. Mech. vol.34, pp.27-37. 
[7] Hossain M.A. and Takhar H.S. (1996): Radiation effect on mixed convection along a vertical plate with uniform surface temperature. - Heat and Mass Transfer, vol.31, pp.243-248.

[8] Rajgopal K., Veena P.H. and Pravin V.K. (2006): Oscillatory motion of an electrically conducting visco-elastic fluid over a stretching sheet in saturated porous medium with suction/blowing. - Mathematical Problem in Engineering, vol.1, pp.1-14.

[9] Singh K.D. (2011): Exact solution of an oscillatory MHD flow in a channel filled with porous medium. - Int. J. Applied Mechanics and Engineering, vol.16, pp.277-283.

[10] Rahman M.M. and Sarkar M.S.A. (2004): Unsteady MHD flowof visco-elastic Oldroyd fluid under time varying body force through a rectangular channel. - Bulletin of Calcutta Mathematical Society, vol.96, pp.463-470.

[11] Singh A.K. and Singh N.P. (1996): MHD flow of a dusty visco-elastic liquid through a porous medium between two inclined parallel plates. - Proceeding of National Academy of Sciences India, vol.66A, pp.143.

[12] Attia Hazem Ali and Karem Mahmoud Ewis (2010): Unsteady MHD Couette flow with heat transfer of a viscoelastic fluid under exponential decaying pressure gradient. - Tankang J. Sci. And Engng., vol.13, pp.359-364.

[13] Choudhary R. and Das U.J. (2012): Heat transfer to MHD oscillatory viscoelastic flow in a channel filled with porous medium. - Physics Research International, doi:101155/2012/879537.

[14] Makinde O.D. and Mhone P.Y. (2005): Heat transfer to MHD oscillatory flow in a channel filled with porous medium. - Rom. Journ. Phys., vol.50, pp.931-938.

[15] Singh K.D. (2012): Viscoelastic mixed convection MHD oscillatory flow through a porous medium filled in a vertical channel. - Int. J. of Phy. And Math. Sci., vol.3, pp.194-205.

[16] Singh K.D. (2013): Effect of slip condition on viscoelastic MHD oscillatory forced convection flow in a vertical channel with heat radiation. - Int. J. of Appl. Mech. and Engng., vol.18, No.4, pp.1237-1248.

[17] Malikov G.K., Lovanov D.L., Malikov K.Y., Lisienko G.V., Viskanta R. and Fedorov A.G. (2001): Direct flame impingement heating for rapid thermal materials processing. - Int. J. Heat and Mass Transfer, vol.44, pp.1751-1758.

[18] Fedorov A.G., Lee K.H. and Viskanta R. (1998): Inverse optimal design of the radiant heating in materials processing and manufacturing. - J. Materials Engineering and Performance, vol.7, pp.719-726.

[19] Lentes F.T. and Siedow N. (1999): Three-dimensional radiative heat transfer in glass cooling processes. - Glass Sci. Technology: Glastechnische Berichte, vol.72, No.6, pp.188-196.

[20] Singh K.D. (1992): Unsteady free convection flow past a hot vertical porous plate with variable temperature. Proc. Indian Natn. Sci. Acad., vol.58, pp.537-544.

[21] Singh K.D. and Khem Chand (2000): Unsteady free convective MHD flow past a vertical porous plate with variable temperature. - Proc. Nat. Acad. Sci. India, vol.70, pp.49-58.

[22] Sumathi K., Anuradha S. and Arunachalam T. (2011): Heat and mass transfer in an unsteady three dimensional mixed convection flow past an infinite vertical porous plate with cosinusoidally fluctuating temperature. International J. Engineering Science and Technology, vol.3, pp.8569-8578.

[23] Kumar R. and Singh K.D. (2011): Unsteady MHD flow of radiating and reacting fluid past a vertical porous plate with cosinusoidally fluctuating temperature. - International J. Appl. Math. and Mech., vol.7, pp.19-35.

[24] Coleman B.D. and Noll W. (1960): An approximation theorem for functional, with applications in continuum mechanics. - Archive for Rational Mechanics and Analysis, vol.6, pp.355-370.

[25] Markovitz H. and Coleman B.D. (1964): Incompressible second order fluids. - Advances in Applied Mechanics, vol.8, pp.69-101.

[26] Raptis A.A., Perdikis C. and Leontitsis K. (2003): Effects of radiation in an optically thin gray gas flowing past a vertical infinite plate in the presence of a magnetic field. - Heat and Mass Transfer, vol.39, pp.771-773. Doi: 10.1007/s00231-002-0317-8. 